\title{
Software pipeline for the analysis of the functional role of nucleotide substitutions in regulatory regions of genes and its testing on polymorphisms associated with obesity
}

\author{
Ekaterina Alekseevna Matrosova \\ Department of Natural Sciences \\ Novosibirsk State University \\ Novosibirsk, Russia \\ MatrosovaEA@bionet.nsc.ru
}

\author{
Vadim Mikhailovich Efimov \\ Laboratory of Molecular Genetic \\ Systems, ICG SB RAS \\ Novosibirsk, Russia \\ Novosibirsk State University \\ Novosibirsk, Russia \\ efimov@bionet.nsc.ru
}

\author{
Elena Vasilevna Ignatieva \\ Laboratory of Evolutionary \\ Bioinformatics and Theoretical \\ Genetics, ICG SB RAS \\ Novosibirsk, Russia \\ eignat@bionet.nsc.ru
}

\begin{abstract}
Genome-wide association studies have shown that approximately $90 \%$ of the nucleotide substitutions associated with diseases are located outside the coding regions of the genes, with about $40 \%$ occurring in regulatory regions. However, the molecular mechanisms by which such SNPs influence incidence of diseases are still poorly understood. In this work, we have developed a software pipeline that allows us to predict the potential effects of nucleotide substitutions on potential transcription factor binding sites (TFBSs). This pipeline integrates and analyzes information obtained from the UCSC Variant Annotation Integrator and PERFECTOS-APE programs, and the dbSNP, Ensembl and HOCOMOCO databases. The operation of the pipeline was tested on two sets of SNPs. All of them were located in the $B D N F$ gene regulatory regions in the vicinity of the SNP rs11030104 associated with elevated body weight. SNPs from the first set were in linkage disequilibrium with rs11030104 and the second set contained random SNPs. We found some differences in the functional characteristics of TFBSs and corresponding transcription factors identified on the basis of the analysis of these two sets of SNPs.
\end{abstract}

Keywords - polymorphism (SNP), transcription factor binding sites (TFBSs), software pipeline, obesity.

\section{Introduction}

Genome-wide association studies have shown that approximately $90 \%$ of the nucleotide substitutions associated with diseases are located outside the coding regions of the genes, with about $40 \%$ occurring in regulatory regions [1]. However, the molecular genetic mechanisms of the influence of the revealed polymorphisms on the development of pathologies have been studied insufficiently. Such genetic variants can be either causative, that is, change the functional activity of regulatory DNA sites (transcription factor binding sites), or they may mark specific haplotypes containing causal SNPs [1-3].

The aim of this work was to create a software pipeline to evaluate the potential effects of nucleotide substitutions on TFBSs. Functionality of the software pipeline was tested on nucleotide variants in regulatory regions of the $B D N F$ gene associated with the development of monogenic forms of obesity [4]. The relevance of the chosen research object is due to the fact that today obesity is an extremely common disease in both developed and developing countries. In addition, obesity may increase the risk of many health problems [5].

\section{Materials and methods}

To write scripts of the software pipeline, the Python was used. Data on polymorphisms was obtained from dbSNP, Ensembl, UCSC table browser, and UCSC Variant Annotation Integrator program output. Weight matrix data and the types of DNA-binding domains of transcription factors (TFs) were obtained from HOCOMOCO [6]. Prediction of the effects of nucleotide substitutions on TFBSs was carried out using the PERFECTOS-APE program using the default settings (P-value cutoff $=5^{*} 10^{-4}$, Fold change cutoff $=4$ ).

The set of SNPs № 1 (case) included 87 SNPs that were in linkage disequilibrium in the European population with the $B D N F$ gene polymorphism rs11030104 associated with increased body weight [7]. The identifiers of these SNPs were extracted from the LDproxy database using the criterion $r^{2}>0.6$. The set of SNPs № 2 (control) included 86 SNPs localized in the vicinity of rs1 1030104 polymorphism but not linked to it and having a minor allele frequency over 0.01 . To compare the results of the analysis of case and control, the statistical chi-squared test and Kullback criterion were used [8]. The p-value threshold for a statistical significance was 0.05 .

\section{Results}

We have developed a software pipeline that includes two original Python scripts that integrate and analyze information obtained from the UCSC Variant Annotation Integrator and PERFECTOS-APE programs, as well as from the dbSNP, Ensembl, and HOCOMOCO databases (Fig. 1). At the output, the pipeline provides information on the effects of nucleotide substitutions on the similarity of a DNA regions with the TFBS weight matrices, on the positions of SNPs in the genome and relative to gene transcription start sites, on the TFs, as well as the p-values characterizing the similarity of pairs of sites with their matrices, its fold change, and some other data. 


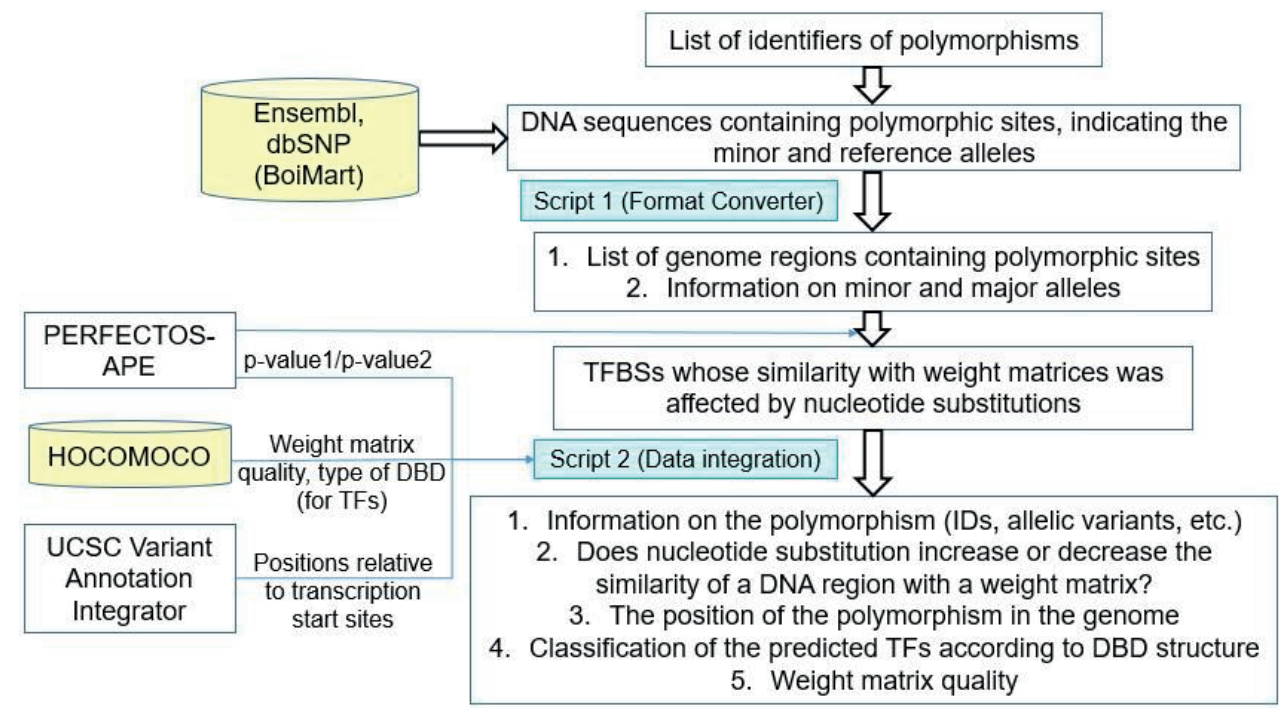

Fig. 1. The scheme of the software pipeline, which allows to analyze the effects of nucleotide substitutions on TFBSs

The software pipeline was tested on the case and the control sets of polymorphisms (see Materials and methods). As a result, 423 and 505 TFBSs were predicted, whose similarity with weight matrices changed by nucleotide substitutions (Table 1). According to the 1000 Genomes Project report [9], for SNPs located in conserved fragments of regulatory regions of the human genome the ratio between the number of nucleotide substitutions damaging the TFBSs and the number of substitutions leading to their occurrence is $4: 1$. Both SNPs sets showed a significant difference in the proportions between positive and negative effects of nucleotide substitutions on TFBSs in comparison with this ratio. In both cases, an approximately equal numbers of both positive and negative effects of nucleotide substitutions on the similarity of TFBSs with the corresponding weight matrices were predicted (Table 1).

TABLE 1 - RESULTS OF ANALYSIS OF SNPS EFFECTS ON TFBSS, OBTAINED BY THE SOFTWARE PIPELINE

\begin{tabular}{|c|c|c|c|c|c|}
\hline \multirow[t]{2}{*}{ SNPs set } & \multirow[t]{2}{*}{$\begin{array}{l}\text { Num- } \\
\text { ber of } \\
\text { SNPs } \\
\text { in the } \\
\text { set }\end{array}$} & \multirow[t]{2}{*}{$\begin{array}{l}\text { Num- } \\
\text { ber of } \\
\text { iden- } \\
\text { tified } \\
\text { TFBSs }\end{array}$} & \multicolumn{2}{|c|}{$\begin{array}{c}\text { Number of TFBSs for } \\
\text { which the similarity } \\
\text { with the weight } \\
\text { matrix has }\end{array}$} & \multirow[t]{2}{*}{$\begin{array}{c}\text { Number of } \\
\text { TFs corres- } \\
\text { ponding to } \\
\text { identified } \\
\text { TFBSs }\end{array}$} \\
\hline & & & increased & decreased & \\
\hline $\begin{array}{c}\text { № } 1 \\
\text { (case) }\end{array}$ & 87 & 423 & 215 & 208 & 246 \\
\hline $\begin{array}{c}\text { № } 2 \\
\text { (control) }\end{array}$ & 86 & 506 & 231 & 275 & 266 \\
\hline
\end{tabular}

It was revealed that the predicted sites corresponded to 246 and 266 transcription factors (Table 1). When comparing the distributions of these TFs sets by the classes of their DNAbinding domains (DBDs), significant differences were identified using the chi-squared test, as well as using Kullback criterion. When comparing the proportion of TFs of a particular DBD class, significant differences were found between sets of TFs for the classes of Basic and Other allalpha-helical types of DBDs. When comparing the proportions of binding sites for specific TFs using Kullback criterion, significant differences were revealed between sets of TFs predicted for the case and the control sets of SNPs.

Similar results were obtained when comparing the sets of predicted TFBSs and TFs for the control SNPs set (on the one hand) and the sets of predicted TFBSs and TFs for SNPs from the case set that have a higher degree of correlation with rs11030104: $r^{2}>0.8$ and $r^{2}>0.9$ (on the other hand) (data not shown).

\section{Conclusion}

We have developed a software pipeline that takes a list of SNP identifiers as an input and allows us to predict the potential effects of nucleotide substitutions on potential transcription factor binding sites. The efficiency of the pipeline was tested on two sets of polymorphisms in the regulatory regions of the $B D N F$ gene associated with obesity.

\section{ACKNOWLEDGMENT} C-01.

Supported by the State Budgeted Project 0324-2019-0040-

\section{REFERENCES}

[1] M. T. Maurano et al., 'Systematic Localization of Common DiseaseAssociated Variation in Regulatory DNA', Science (80-. )., vol. 337, no. 6099, pp. 1190-1195, Sep. 2012.

[2] J. M. Seddon, K. T. Berggren, and L. M. Fleeman, 'Evolutionary history of DLA class II haplotypes in canine diabetes mellitus through single nucleotide polymorphism genotyping', Tissue Antigens, vol. 75, no. 3, pp. 218-226, Mar. 2010.

[3] Y. Zhang, M. Manjunath, S. Zhang, D. Chasman, S. Roy, and J. S. Song, 'Integrative Genomic Analysis Predicts Causative Cis Regulatory Mechanisms of the Breast Cancer-Associated Genetic Variant rs4415084', Cancer Res., vol. 78, no. 7, pp. 1579-1591, Apr. 2018 .

[4] J. Gray et al., 'Hyperphagia, severe obesity, impaired cognitive function, and hyperactivity associated with functional loss of one copy of the brain-derived neurotrophic factor (BDNF) gene.', Diabetes, vol. 55, no. 12, pp. 3366-71, Dec. 2006.

[5] K. R. Fontaine, D. T. Redden, C. Wang, A. O. Westfall, and D. B. Allison, 'Years of life lost due to obesity', JAMA, vol. 289, pp. 187193, 2003.

[6] I. V Kulakovskiy et al., 'HOCOMOCO: towards a complete collection of transcription factor binding models for human and mouse via largescale ChIP-Seq analysis', Nucleic Acids Res., vol. 46, no. D1, pp. D252-D259, Jan. 2018.

[7] A. E. Locke et al., 'Genetic studies of body mass index yield new insights for obesity biology', Nature, vol. 518, no. 7538, pp. 197-206, Feb. 2015.

[8] S. Kullback, Information theory and statistics. Peter Smith, 1978.

[9] 1000 Genomes Project Consortium et al., 'An integrated map of genetic variation from 1,092 human genomes', Nature, vol. 491, no. 7422, pp. 56-65, Nov. 2012. 